\title{
IMPROVED MATRIX PENCIL METHODS
}

\author{
Biao Lu ${ }^{1}$, Dong Wei ${ }^{2}$, Brian L. Evans ${ }^{1}$, and Alan C. Bovik ${ }^{1}$ \\ ${ }^{1}$ Dept. of Electrical and Computer Engineering \\ The University of Texas at Austin, Austin, TX 78712-1084 \\ $\{$ blu, bevans, bovik\}@ece.utexas. edu \\ ${ }^{2}$ Dept. of Electrical and Computer Engineering, \\ Drexel University, Philadelphia, PA 19104 \\ wei@ece.drexel.edu
}

\begin{abstract}
We study the problem of estimating signal parameters from a noisy data sequence containing superimposed damped sinusoids. We propose three novel methods by combining the reduced-rank Hankel approximation and the Matrix Pencil method. We demonstrate that two of the proposed methods significantly outperform both the original Matrix Pencil method and the modified Kumaresan-Tufts method, especially at low signal-tonoise ratio.
\end{abstract}

\section{INTRODUCTION}

Estimating signal parameters from a noisy superimposed damped sinusoidal data sequence has found many diverse applications, such as determination of directionof-arrival plane waves at a uniform linear array of sensors and estimation of Doppler frequencies [1-6]. An effective way to combat observation noise and obtain accurate estimates is to exploit the properties possessed by the unknown signal. For a data sequence consisting of superimposed damped sinusoids, the data matrix in the corresponding matrix prediction equation enjoys both a rank-deficient property and a Hankel structure.

The Kumaresan-Tufts (KT) method exploits the structure of the backward linear prediction equations satisfied by the underlying signal to estimate signal parameters, and applies a reduced-rank approximation based on singular value decomposition (SVD) to mitigate noise effects [1]. However, such an approximation takes into account only the rank-deficient property

This work was supported by grants from Schlumberger Austin Research, Southwestern Bell Technology Resources, Inc., the Defense Advanced Research Projects Agency (DARPA) and the US Army in the Composite CAD Program under DARPA Grant DAAB07-97-C-J007, and an NSF CAREER Award under grant MIP-9702707. of the signal matrix and does not preserve its Hankel structure. As a consequence, the resulting performance breaks down at low signal-to-noise ratio (SNR). Hua and Sarkar developed the Matrix Pencil (MP) method [2] which is an efficient method that achieves better estimation performance than the KT method. It exploits the structure of a matrix pencil of the underlying signal and also makes use of the SVD-based reduced-rank approximation to suppress the noise. Like the KT method, the MP method ignores the Hankel structure of the matrix pencil and suffers from a degradation of estimation accuracy at low SNR. Cadzow proposed a signal enhancement algorithm for estimating the signal parameters of undamped sinusoids [3], [4], which exploits both the rank-deficient and ToeplitzHankel properties of the signal matrix. Such an algorithm was extended to improve the KT method for damped sinusoids, where an iterative reduced-rank Hankel approximation (RRHA) was developed [4], [5] and shown to yield better performance than the original KT method.

We apply the concept of RRHA to improve the MP method. We propose three novel methods by combining the RRHA and original MP method, and compare them with the original MP method and the modified KT (MKT) method proposed in [4] and [5]. We demonstrate that two of the proposed methods outperform both the original MP method and the MKT method in terms of estimation accuracy.

Section 2 reviews the MP method and RRHA concept. Section 3 presents three RRHA-based modified MP methods. Razavilar, Li, and Liu [6] introduce a method similar to our modified matrix pencil method \#3 described in Section 3.3. However, their method can be used only for square data matrices whereas our three new methods can be applied to rectangular data matrices. Section 4 demonstrates our simulation re- 
sults and Section 5 concludes the paper.

\section{BACKGROUND}

We consider the problem of estimating signal parameters from a noisy observed data sequence of $K$ samples

$$
r_{k}=s_{k}+n_{k},
$$

where the noise term $n_{k}$ is a complex white Gaussian random process, and the noise-free signal $s_{k}$ is given by

$$
s_{k}=\sum_{m=1}^{M} a_{m} e^{\left(-d_{m}+j 2 \pi f_{m}\right) k}
$$

for $k=0,1, \ldots, K-1$, where $M$ is the number of exponentially damped sinusoids, and the $a_{m}, d_{m}$, and $f_{m}$ terms represent the complex amplitudes, damping factors, and frequencies, respectively, which are the unknown signal parameters to be estimated. The set of amplitude terms $\left\{a_{m}\right\}$ can be estimated by solving a linear least squares problem if all of the other parameters are known. The parameter $M$ can be estimated via various methods (e.g., [1] and [7]). In this paper, we shall focus on estimation of the $\left\{d_{m}\right\}$ and $\left\{f_{m}\right\}$ terms.

\subsection{Matrix Pencil Method}

Define subscripted vectors as

$$
\boldsymbol{r}_{l}=\left[\begin{array}{llll}
r_{l}^{*} & r_{l+1}^{*} & \ldots & r_{K-L+l-1}^{*}
\end{array}\right]^{T}
$$

for $l=0,1, \ldots, L$ where the superscripts ${ }^{*}$ and ${ }^{T}$ denote the complex conjugate and matrix transpose, respectively. We form three data matrices from the observed data sequence $\left\{r_{k}\right\}_{k=0}^{K-1}$ :

$$
\begin{aligned}
\boldsymbol{R} & =\left[\begin{array}{llll}
\boldsymbol{r}_{0} & \boldsymbol{r}_{1} & \ldots & \boldsymbol{r}_{L}
\end{array}\right]_{(K-L) \times(L+1)} \\
\boldsymbol{R}^{0} & =\left[\begin{array}{llll}
\boldsymbol{r}_{0} & \boldsymbol{r}_{1} & \ldots & \boldsymbol{r}_{L-1}
\end{array}\right]_{(K-L) \times L} \\
\boldsymbol{R}^{1} & =\left[\begin{array}{llll}
\boldsymbol{r}_{1} & \boldsymbol{r}_{2} & \ldots & \boldsymbol{r}_{L}
\end{array}\right]_{(K-L) \times L}
\end{aligned}
$$

$L$ is called the pencil parameter and $\boldsymbol{R}$ is referred to as the master matrix. The signal matrices $\boldsymbol{S}, \boldsymbol{S}^{0}$, and $\boldsymbol{S}^{1}$ are defined similarly from $\left\{s_{k}\right\}_{k=0}^{K-1}$. All three matrices $\boldsymbol{S}, \boldsymbol{S}^{0}$, and $\boldsymbol{S}^{1}$ have rank $M$ and Hankel structure. $\left\{e^{d_{m}-j 2 \pi f_{m}}\right\}_{m=1}^{M}$ is the set of $M$ non-zero eigenvalues of $\left(\boldsymbol{S}^{0}\right)^{\dagger} \boldsymbol{S}^{1}$, where ${ }^{\dagger}$ denotes the pseudoinverse [2].

In the presence of noise, the matrices $\boldsymbol{R}, \boldsymbol{R}^{0}$, and $\boldsymbol{R}^{1}$ are full rank but still have Hankel structure. Hua and Sarkar [2] perform an SVD and its rank- $M$ truncation of the $M$ largest singular values on the noisy data matrices $\boldsymbol{R}^{0}$ and $\boldsymbol{R}^{1}$ to reduce the effect of noise. For a given rank- $L$ matrix $\boldsymbol{X}$, we define the rank- $M$ $(M \leq L)$ approximation operator $\mathcal{L}$ as

$$
\mathcal{L}\{\boldsymbol{X}\}=\sum_{m=1}^{M} \sigma_{m} \boldsymbol{u}_{m} \boldsymbol{v}_{m}^{H}
$$

where $\left\{\sigma_{1}, \sigma_{2}, \cdots, \sigma_{M}\right\}$ is the set of the $M$ largest singular values of $\boldsymbol{X}$, the $\boldsymbol{u}_{m}$ and $\boldsymbol{v}_{m}$ terms are the corresponding left and right singular vectors, respectively, and the superscript ${ }^{H}$ is the matrix conjugate transpose. We compute $\mathcal{L}\left\{\boldsymbol{R}^{i}\right\}$, which is a rank-M approximation of $\boldsymbol{S}^{i}$ for $i=0,1$. Then, we estimate $\left\{d_{m}\right\}$ and $\left\{f_{m}\right\}$ by computing the $M$ non-zero eigenvalues of matrix $\left(\mathcal{L}\left\{\boldsymbol{R}^{0}\right\}\right)^{\dagger} \mathcal{L}\left\{\boldsymbol{R}^{1}\right\}$. The MP method follows:

1. Compute $\widetilde{\boldsymbol{S}}^{0}=\mathcal{L}\left\{\boldsymbol{R}^{0}\right\}$

2. Compute $\widetilde{\boldsymbol{S}}^{1}=\mathcal{L}\left\{\boldsymbol{R}^{1}\right\}$

3. Compute the $M$ non-zero eigenvalues of $\left(\widetilde{\boldsymbol{S}}^{0}\right)^{\dagger} \widetilde{\boldsymbol{S}}^{1}$.

In general, the rank- $M$ matrices $\mathcal{L}\left\{\boldsymbol{R}^{0}\right\}$ and $\mathcal{L}\left\{\boldsymbol{R}^{1}\right\}$ do not have Hankel structure, which may result in highly biased estimates of the parameters, esp. at low SNR.

\subsection{Reduced-Rank Hankel Approximation}

For a given $P \times Q$ matrix $\boldsymbol{X}$, we define the Hankel approximation operator $\mathcal{H}$ as

$$
\boldsymbol{Y}=\mathcal{H}\{\boldsymbol{X}\}
$$

in which the $(p, q)$-th element of $\boldsymbol{Y}$ is given by

$$
y_{p, q}=\frac{1}{\left|\Lambda_{p+q}\right|} \sum_{\left(p^{\prime}, q^{\prime}\right) \in \Lambda_{p+q}} x_{p^{\prime}, q^{\prime}}
$$

for $p=0,1, \ldots, P-1$, and $q=0,1, \ldots, Q-1$, where $\Lambda_{t}$ denotes the set of indices corresponding to the $t^{\text {th }}$ anti-diagonal in a matrix, i.e., $\Lambda_{t}=\left\{\left(p^{\prime}, q^{\prime}\right): 0 \leq p^{\prime} \leq\right.$ $\left.P-1,0 \leq q^{\prime} \leq Q-1, p^{\prime}+q^{\prime}=t\right\}$, and $\left|\Lambda_{t}\right|$ denotes the cardinality of the set $\Lambda_{t}$. In general, the operator $\mathcal{L}$ does not preserve the Hankel property, and the operator $\mathcal{H}$ does not preserve the rank-deficient property. Since there is a one-to-one correspondence between a data sequence consisting of superimposed damped sinusoids and a rank-deficient Hankel matrix [5], it is desirable to maintain both the rank-deficient and Hankel properties of the data matrix. Thus, we define $\mathcal{J}$ to be the reduced-rank Hankel approximation (RRHA) operator that generates a matrix having both the rankdeficient and Hankel properties. Since no analytic form for the operator $\mathcal{J}$ exists to date, an iterative algorithm is used to approximate $\mathcal{J}$ [5]:

$$
\begin{aligned}
\mathcal{J}\{\boldsymbol{X}\} & =(\mathcal{H L})^{\infty}\{\boldsymbol{X}\}=\lim _{L \rightarrow \infty}(\mathcal{H L})^{L}\{\boldsymbol{X}\} \\
& =\lim _{L \rightarrow \infty} \underbrace{(\mathcal{H L} \cdots(\mathcal{H} \mathcal{L}}_{L}(\mathcal{H} \mathcal{L}\{\boldsymbol{X}\})) \cdots) .
\end{aligned}
$$

Cadzow shows that such an iteration converges for the data matrix $\boldsymbol{R}$ associated with the superimposed damped sinusoids buried in noise [3]. Theoretically, the matrix 
$\mathcal{J}\{\boldsymbol{R}\}$ possesses both the Hankel and rank-deficient properties and results in a better approximation of $\boldsymbol{S}$ than the reduced-rank but non-Hankel matrix $\mathcal{L}\{\boldsymbol{R}\}$.

However, in reality only a finite number of iterations can be implemented (thus, a stopping criterion is needed in practice) so that the resulting matrix is either roughly rank-deficient or roughly Hankel. Usually, we choose to force the matrix to be exactly rank-deficient and approximately Hankel in order to make the subsequent steps (e.g., finding non-zero eigenvalues) easier.

\section{MODIFIED MATRIX PENCIL METHODS}

Since three matrices, $\boldsymbol{S}, \boldsymbol{S}^{0}$, and $\boldsymbol{S}^{1}$, are involved in the MP method, it would be desirable to make use of rank-deficient and Hankel properties of all three matrices. Next, we shall develop three modified MP methods classified by the properties they exploit.

\subsection{Method Matrix Pencil Method 1 (MMP1)}

A straightforward modification of the original matrix pencil method is to replace the reduced-rank approximation of $\boldsymbol{R}^{0}$ and $\boldsymbol{R}^{1}$ by the RRHA of the two matrices so that the Hankel structure of $\boldsymbol{S}^{0}$ and $\boldsymbol{S}^{1}$ is exploited, too. Fig. 1 gives the steps to implement this method.

According to Theorem 1 in [5], we infer that during each iteration of the RRHA, the preservation of the Hankel structure leads to a better approximation of the true signal matrices $\boldsymbol{S}^{0}$ and $\boldsymbol{S}^{1}$; i.e., in the $l$ th iteration,

$$
\left\|(\mathcal{H L})^{l}\left\{\boldsymbol{R}^{i}\right\}-\boldsymbol{S}^{i}\right\|_{F} \leq\left\|\mathcal{L}(\mathcal{H L})^{l-1}\left\{\boldsymbol{R}^{i}\right\}-\boldsymbol{S}^{i}\right\|_{F}
$$

for $i=0,1$, where $\|\cdot\|_{F}$ denotes the Frobenius norm: $\|\boldsymbol{X}\|_{F}=\left(\sum_{p=0}^{P-1} \sum_{q=0}^{Q-1}\left|x_{p, q}\right|^{2}\right)^{1 / 2}$ for a $P \times Q$ matrix $\boldsymbol{X}$. Like the original MP method, a disadvantage of the MMP1 method is that, since the two matrices $\boldsymbol{R}^{0}$ and $\boldsymbol{R}^{1}$ are processed independently, the properties of the matrix $\boldsymbol{S}$ are ignored. Therefore, in general, $\widetilde{\boldsymbol{S}}^{0}$ and $\widetilde{\boldsymbol{S}}^{1}$ in MMP1 method do not correspond to any valid reduced-rank and Hankel approximation of $\boldsymbol{S}$. This drawback can yield significantly biased estimates when the two signals corresponding to $\widetilde{\boldsymbol{S}}^{0}$ and $\widetilde{\boldsymbol{S}}^{1}$ are very different from each other.

\begin{tabular}{l} 
1. Compute $\widetilde{\boldsymbol{S}}^{0}=\mathcal{J}\left\{\boldsymbol{R}^{0}\right\}$ \\
2. Compute $\widetilde{\boldsymbol{S}}^{1}=\mathcal{J}\left\{\boldsymbol{R}^{1}\right\}$ \\
3. Compute the $M$ non-zero eigenvalues of $\left(\widetilde{\boldsymbol{S}}^{0}\right)^{\dagger} \widetilde{\boldsymbol{S}}^{1}$ \\
\hline
\end{tabular}

Figure 1: Modified Matrix Pencil Method 1 (MMP1)

\subsection{Modified Matrix Pencil Method 2 (MMP2)}

To avoid the highly biased estimates caused by the independent processing of $\boldsymbol{R}^{0}$ and $\boldsymbol{R}^{1}$, we consider the rank-deficient property and/or the Hankel property possessed by $\boldsymbol{S}$ as well; i.e., we need to process the two matrices $\boldsymbol{R}^{0}$ and $\boldsymbol{R}^{1}$ jointly. The key idea is to modify the RRHA operator by performing the Hankel operator on the two rank-reduced matrices simultaneously rather than individually so that the corresponding master matrix will also possess the Hankel structure. We extend the operator $\mathcal{H}$ to a pair of matrices $\left\{\boldsymbol{Y}^{0}, \boldsymbol{Y}^{1}\right\}=\mathcal{H}\left\{\boldsymbol{X}^{0}, \boldsymbol{X}^{1}\right\}$. The elements of $\boldsymbol{Y}^{0}$ and $\boldsymbol{Y}^{1}$ are

$$
\begin{aligned}
& y_{p, q}^{0}=\frac{\sum_{\left(p^{\prime}, q^{\prime}\right) \in \Lambda_{p+q}} x_{p^{\prime}, q^{\prime}}^{0}+\sum_{\left(p^{\prime}, q^{\prime}\right) \in \Lambda_{p+q-1}} x_{p^{\prime}, q^{\prime}}^{1}}{\left|\Lambda_{p+q}\right|+\left|\Lambda_{p+q-1}\right|} \\
& y_{p, q}^{1}=\frac{\sum_{\left(p^{\prime}, q^{\prime}\right) \in \Lambda_{p+q+1}} x_{p^{\prime}, q^{\prime}}^{0}+\sum_{\left(p^{\prime}, q^{\prime}\right) \in \Lambda_{p+q}} x_{p^{\prime}, q^{\prime}}^{1}}{\left|\Lambda_{p+q+1}\right|+\left|\Lambda_{p+q}\right|}
\end{aligned}
$$

for $p=0,1, \ldots, P-1$, and $q=0,1, \ldots, Q-1$. We summarize the resulting "MMP2" method in Figure 2:

The proof of the convergence of such an iterative algorithm remains open. However, it always approximately converges within a few iterations in our simulations over a wide range of SNRs. Each iteration better approximates $\boldsymbol{S}^{0}$ and $\boldsymbol{S}^{1}$. In the $l$ th iteration,

$$
\sum_{i=0}^{1}\left\|(\mathcal{H L})^{l}\left\{\boldsymbol{R}^{i}\right\}-\boldsymbol{S}^{i}\right\|_{F}^{2} \leq \sum_{i=0}^{1}\left\|\mathcal{L}(\mathcal{H} \mathcal{L})^{l-1}\left\{\boldsymbol{R}^{i}\right\}-\boldsymbol{S}^{i}\right\|_{F}^{2}
$$

Even though the improvement in the matrix approximation described in (4) seems weaker than the one given in (3), we expect that the MMP2 method yields more accurate estimates of signal parameters than the MMP1 method, especially at low SNR. The better performance results because the MMP2 method makes use of the relationship between $\boldsymbol{S}^{0}$ and $\boldsymbol{S}^{1}$.

\subsection{Modified Matrix Pencil Method 3 (MMP3)}

Another way to exploit the properties of the three matrices $\boldsymbol{S}, \boldsymbol{S}^{0}$, and $\boldsymbol{S}^{1}$ jointly, is to perform the RRHA
1. Let $\widetilde{\boldsymbol{S}}^{0}=\boldsymbol{R}^{0}$ and $\widetilde{\boldsymbol{S}}^{1}=\boldsymbol{R}^{1}$
2. Compute $\left\{\widehat{\boldsymbol{S}}^{0}, \widehat{\boldsymbol{S}}^{1}\right\}=\mathcal{H}\left\{\widetilde{\boldsymbol{S}}^{0}, \widetilde{\boldsymbol{S}}^{1}\right\}$
3. Compute $\widetilde{\boldsymbol{S}}^{0}=\mathcal{L}\left\{\widehat{\boldsymbol{S}}^{0}\right\}$
4. Compute $\widetilde{\boldsymbol{S}}^{1}=\mathcal{L}\left\{\widehat{\boldsymbol{S}}^{1}\right\}$
5. Go to Step 2 unless the stop criterion is satisfied
6. Compute the $M$ non-zero eigenvalues of $\left(\widetilde{\boldsymbol{S}}^{0}\right)^{\dagger} \widetilde{\boldsymbol{S}}^{1}$.

Figure 2: Modified Matrix Pencil Method 2 (MMP2) 


\begin{tabular}{l} 
1. Compute $\widehat{\boldsymbol{S}}=\mathcal{J}\{\boldsymbol{R}\}$ \\
2. Form $\widehat{\boldsymbol{S}}^{0}$ and $\widehat{\boldsymbol{S}}^{1}$ from $\widehat{\boldsymbol{S}}$ \\
3. Compute $\widetilde{\boldsymbol{S}}^{0}=\mathcal{L}\left\{\widehat{\boldsymbol{S}}^{0}\right\}$ \\
4. Compute $\widetilde{\boldsymbol{S}}^{1}=\mathcal{L}\left\{\widehat{\boldsymbol{S}}^{1}\right\}$ \\
5. Compute the $M$ non-zero eigenvalues of $\left(\widetilde{\boldsymbol{S}}^{0}{ }^{\dagger} \widetilde{\boldsymbol{S}}^{1}\right.$. \\
\hline
\end{tabular}

Figure 3: Modified Matrix Pencil Method \#3 (MMP3)

on the master data matrix $\boldsymbol{R}$ directly. Since in theory there exists a unique exponential data sequence corresponding to a rank-deficient Hankel matrix $\widehat{\boldsymbol{S}}$, the matrices $\widehat{\boldsymbol{S}}^{0}$ and $\widehat{\boldsymbol{S}}^{1}$ are both rank-deficient and Hankel, too. Therefore, in theory, the two properties are satisfied by all the three matrices. We call such a method the "MMP3" method, which may be viewed as applying a preprocessing procedure to the original MP method. It can be implemented according to Figure 3:

The MMP3 method achieves a better approximation of the true master matrix $\boldsymbol{S}$; i.e., in the $\boldsymbol{l}$ th iteration,

$$
\left\|(\mathcal{H L})^{l}\{\boldsymbol{R}\}-\boldsymbol{S}\right\|_{F} \leq\left\|\mathcal{L}(\mathcal{H} \mathcal{L})^{l-1}\{\boldsymbol{R}\}-\boldsymbol{S}\right\|_{F} .
$$

Since in practice $\widehat{\boldsymbol{S}}$ is only approximately Hankel, $\widehat{\boldsymbol{S}}^{0}$ and $\widehat{\boldsymbol{S}}^{1}$ are neither exactly rank-deficient nor exactly Hankel. However, because of the reduced-rank operator embedded in the subsequent MP method, the rankdeficiency of $\widehat{\boldsymbol{S}}^{0}$ and $\widehat{\boldsymbol{S}}^{1}$ can be eventually achieved. Razavilar, Li, and Liu [6] propose such a method. However, their method requires the data matrices to be square in order to obtain the best performance. This requirement is not necessary since Hua and Sarkar point out that any values satisfying $K / 3 \leq L \leq 2 K / 3$ are good choices [2].

\section{SIMULATION RESULTS}

In our computer simulations, we use the same example as the one in [5] to compare the estimation performance of the MKT method, the MP method, and our three modified MP methods. We choose $M=2, K=25$, $L=17, a_{1}=a_{2}=1, d_{1}=0.2, d_{2}=0.1, f_{1}=0.42$, and $f_{2}=0.52$. The SNR in $\mathrm{dB}$ is defined as SNR = $-10 \log _{10}\left(2 \sigma_{n}^{2}\right)$, where $\sigma_{n}^{2}$ is the variance of the zeromean complex white Gaussian noise process $n_{k}$. We use mean square error (MSE) to assess the estimation accuracy of the parameters $d_{1}, d_{2}, f_{1}$, and $f_{2}$. From each parameter estimate, Figure 4 shows the MSE vs. SNR plots of the five methods as well as the CramerRao Bound (CRB). Each data point is measured based on 500 independent runs of the random noise process.
From Figure 4, all five methods yield about the same results at high SNRs, but quite different results at low SNRs. The MMP1 method achieves comparable performance in estimating the frequencies as the MP method, and performs worse in estimating the damping factors. This may be caused by the negligence of the rank-deficient and Hankel properties possessed by the master matrix. Both MMP2 and MMP3 methods performed significantly better than the MP method in estimating $f_{2}, d_{1}$, and $d_{2}$, and as well as the latter in estimating $f_{1}$. This is consistent with our analysis. On the other hand, both the MMP2 and MMP3 methods achieve performance comparable to the MKT method in terms of the estimation accuracy of the damping factors, and significantly outperform the MKT method in the estimation accuracy of the frequencies.

\section{CONCLUSIONS}

We have presented a comparative study of three modified versions of the MP method. We conclude that both the MMP2 and MMP3 methods achieve significantly better performance than the original MP method, and also outperform the MKT method. The improvement in performance increases as SNR decreases.

In addition, our modified MP methods can be easily extended to the forward-and-backward MP method [2] for estimating parameters of undamped sinusoids. The effect of noise in the data matrices can be attenuated by using a reduced-rank joint Toeplitz-Hankel approximation, which was developed in [4] for improving the Tufts-Kumaresan method [8].

\section{Appendix}

Let $\tilde{\boldsymbol{S}}^{i}=\mathcal{L}(\mathcal{H L})^{l-1}\left\{\boldsymbol{R}^{i}\right\}$ and $\widehat{\boldsymbol{S}}^{i}=(\mathcal{H} \mathcal{L})^{l}\left\{\boldsymbol{R}^{i}\right\}$, for $i=0,1$. We consider the approximation of a pair of diagonals in $\boldsymbol{S}^{0}$ and $\boldsymbol{S}^{1}$ given by $\left\{s_{p, q}^{0}:(p, q) \in \Lambda_{z}\right\}$ and $\left\{s_{p, q}^{1}:(p, q) \in\right.$ $\left.\Lambda_{z-1}\right\}$, respectively,

$$
\begin{aligned}
& \sum_{(p, q) \in \Lambda_{z}}\left|s_{z}^{*}-\widetilde{s}_{p, q}^{0}\right|^{2}+\sum_{(p, q) \in \Lambda_{z-1}}\left|s_{z}^{*}-\widetilde{s}_{p, q}^{1}\right|^{2} \\
= & \sum_{(p, q) \in \Lambda_{z}}\left(\left|s_{z}^{*}-\widehat{s}_{p, q}^{0}\right|^{2}+\left|\widehat{s}_{p, q}^{0}-\widetilde{s}_{p, q}^{0}\right|^{2}\right. \\
& \left.-2 \Re\left\{\left(s_{z}^{*}-\widehat{s}_{p, q}^{0}\right)\left(\widehat{s}_{p, q}^{0}-\widehat{s}_{p, q}^{0}\right)^{*}\right\}\right) \\
& +\sum_{(p, q) \in \Lambda_{z-1}}\left(\left|s_{z}^{*}-\widehat{s}_{p, q}^{1}\right|^{2}+\left|\widehat{s}_{p, q}^{1}-\widetilde{s}_{p, q}^{1}\right|^{2}\right. \\
= & \sum_{(p, q) \in \Lambda_{z}}\left(\left|s_{z}^{*}-\widehat{s}_{p, q}^{0}\right|^{2}+\left|\widehat{s}_{p, q}^{0}-\widehat{s}_{p, q}^{0}\right|^{2}\right) \\
& +\sum_{(p, q) \in \Lambda_{z-1}}\left(\left|s_{z}^{*}-\widehat{s}_{p, q}^{1}\right|^{2}+\left|\widehat{s}_{p, q}^{1}-\widetilde{s}_{p, q}^{1}\right|^{2}\right)
\end{aligned}
$$




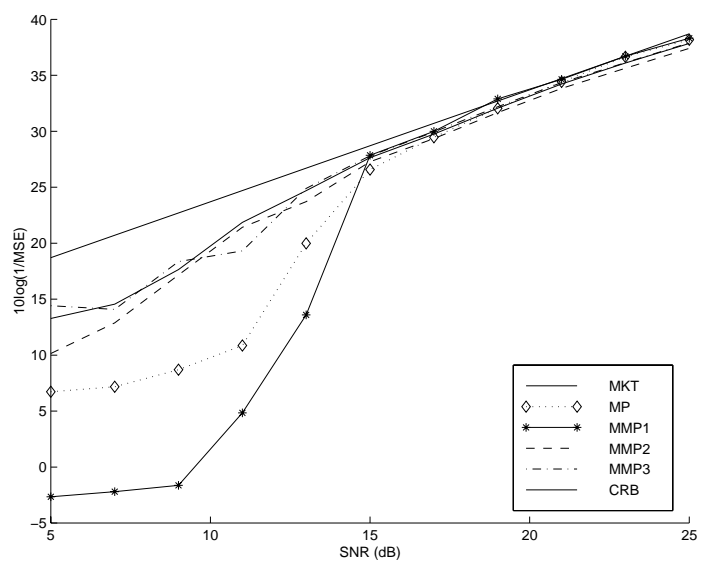

(a)

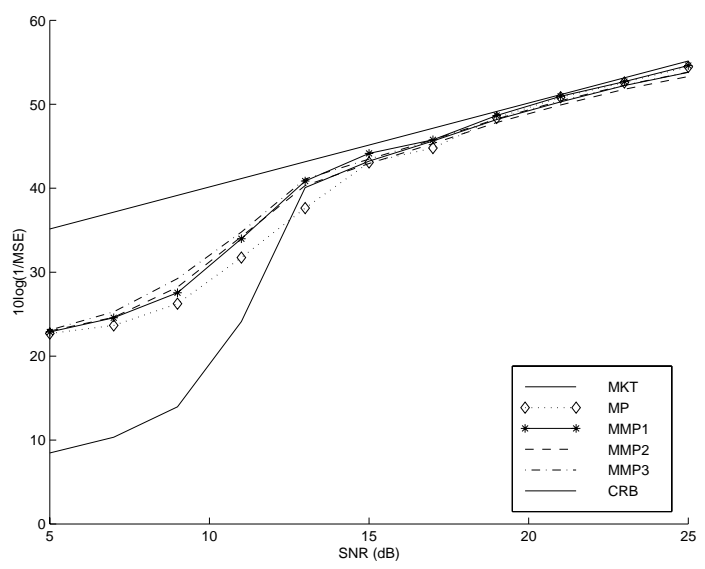

(c)

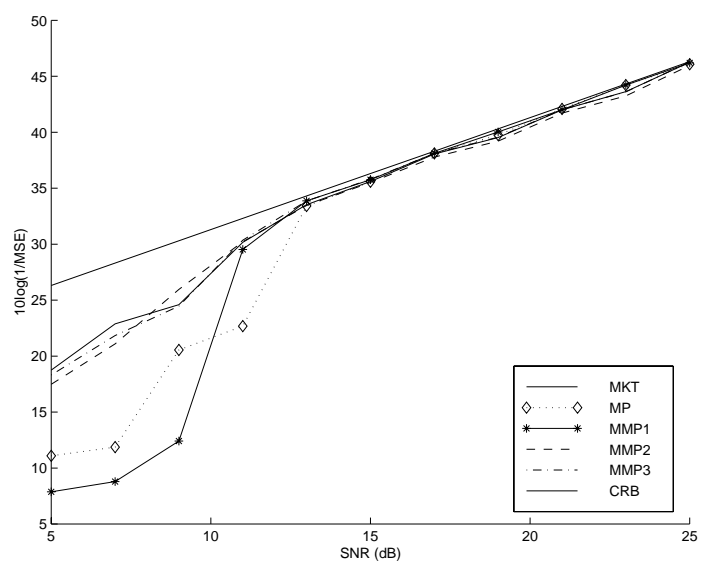

(b)

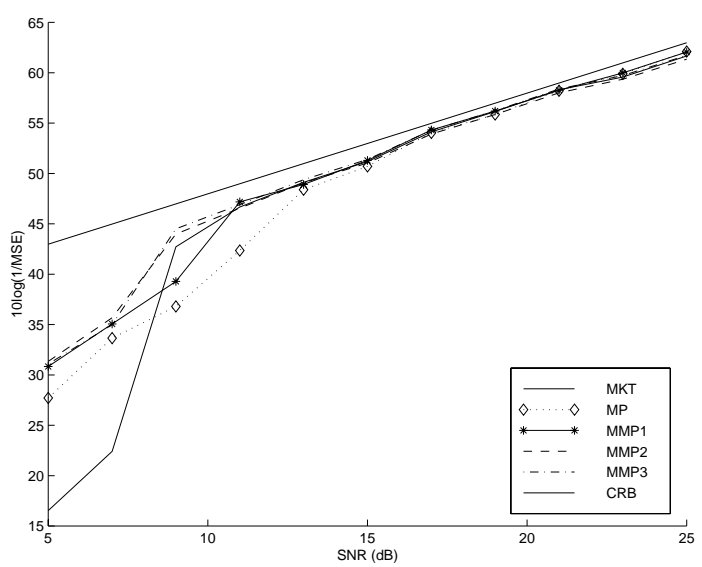

(d)

Figure 4: Performance of the five methods based on 500 trials in estimating: (a) $d_{1}$, (b) $d_{2}$, (c) $f_{1}$, and (d) $f_{2}$.

$\geq \sum_{(p, q) \in \Lambda_{z}}\left|s_{z}^{*}-\hat{s}_{p, q}^{0}\right|^{2}+\sum_{(p, q) \in \Lambda_{z-1}}\left|s_{z}^{*}-\widehat{s}_{p, q}^{1}\right|^{2}$

Considering all the corresponding pairs of diagonals in $\boldsymbol{S}^{0}$ and $\boldsymbol{S}^{1}$, we have proved (4).

\section{REFERENCES}

[1] R. Kumaresan and D. W. Tufts, "Estimating the parameters of exponentially damped sinusoids and polezero modeling in noise," IEEE Trans. Acoust. Speech Signal Processing, vol. 30, pp. 833-840, Dec. 1982.

[2] Y. Hua and T. K. Sarkar, "Matrix pencil method for estimating parameters of exponentially damped/undamped sinusoids in noise," IEEE Trans. Acoust. Speech Signal Processing, vol. 38, pp. 814-824, May 1990.

[3] J. A. Cadzow, "Signal enhancement: A composite property mapping algorithm," IEEE Trans. Acoust. Speech Signal Processing, vol. 36, pp. 49-62, Jan. 1988.
[4] J. A. Cadzow, Y. Sun, and G. Xu, "Detection of multiple sinusoids in white noise: A signal enhancement approach," in SVD and Signal Processing (E. F. Deprettere, ed.), pp. 171-186, North-Holland: Elsevier, 1988.

[5] Y. Li, K. J. R. Liu, and J. Razavilar, "A parameter estimation scheme for damped sinusoidal signals based on low-rank Hankel approximation," IEEE Trans. Signal Processing, vol. 45, pp. 481-486, Feb. 1997.

[6] J. Razavilar, Y. Li, and K. J. R. Liu, "Spectral estimation based on structured low rank matrix pencil," in Proc. IEEE Int. Conf. Acoust. Speech Signal Processing, vol. 5, (Atlanta, GA), pp. 2503-2506, May 1996.

[7] V. Reddy and L. S. Biradar, "SVD-based information theoretic criteria for detection of the number of damped/undamped sinusoids and their performance analysis," IEEE Trans. Signal Processing, vol. 41, pp. 2872-2881, Sept. 1993.

[8] D. W. Tufts and R. Kumaresan, "Estimation of frequencies of multiple sinusoids: Making linear prediction perform like maximum likelihood," Proc. IEEE, vol. 70, pp. 975-989, Sept. 1982. 